\title{
Video Article \\ Exfoliation and Analysis of Large-area, Air-Sensitive Two-Dimensional Materials
}

\author{
Josh P. Thompson ${ }^{1}$, M. Hasan Doha ${ }^{1}$, Peter Murphy ${ }^{1,2}$, Jin Hu ${ }^{1,3}$, Hugh O.H. Churchill \\ ${ }^{1}$ Department of Physics, University of Arkansas \\ ${ }^{2}$ Department of Physics, SUNY Geneseo \\ ${ }^{3}$ Institute for Nanoscale Science and Engineering, University of Arkansas
}

Correspondence to: Hugh O.H. Churchill at hchurch@uark.edu

URL: https://www.jove.com/video/58693

DOI: doi:10.3791/58693

Keywords: Retraction, Issue 143, Two-dimensional materials, black phosphorus, chromium triiodide, oxidation, hydration, air sensitivity

Date Published: 1/5/2019

Citation: Thompson, J.P., Doha, M.H., Murphy, P., Hu, J., Churchill, H.O. Exfoliation and Analysis of Large-area, Air-Sensitive Two-Dimensional Materials. J. Vis. Exp. (143), e58693, doi:10.3791/58693 (2019).

\section{Abstract}

We describe methods for producing and analyzing large, thin flakes of air-sensitive two-dimensional materials. Thin flakes of layered or van der Waals crystals are produced using mechanical exfoliation, in which layers are peeled off a bulk crystal using adhesive tape. This method produces high-quality flakes, but they are often small and can be hard to find, particularly for materials with relatively high cleavage energies such as black phosphorus. By heating the substrate and the tape, two-dimensional material adhesion to the substrate is promoted, and the flake yield can be increased by up to a factor of ten. After exfoliation, it is necessary to image or otherwise analyze these flakes but some twodimensional materials are sensitive to oxygen or water and will degrade when exposed air. We have designed and tested a hermetic transfer cell to temporarily maintain the inert environment of a glovebox so that air-sensitive flakes can be imaged and analyzed with minimal degradation. The compact design of the transfer cell is such that optical analysis of sensitive materials can be performed outside of a glovebox without specialized equipment or modifications to existing equipment.

\section{Video Link}

The video component of this article can be found at https://www.jove.com/video/58693/

\section{Introduction}

Various layered materials that can be exfoliated down to a single atomic layer have generated interest across a broad range of fields. However, investigation and application of many of these materials is complicated by the fact that they are unstable in air and quickly oxidize or hydrate when exposed. For example; black phosphorus is a semiconductor with a tunable direct band gap, high mobility and anisotropic optical and electrical properties ${ }^{1,2,3,4,5}$ but is unstable in air and will deteriorate in less than an hour ${ }^{6,7}$ due to interactions with oxygen ${ }^{8}$. Crl ${ }_{3}$ has been recently $^{1}$ shown to exhibit two-dimensional ferromagnetism ${ }^{9,10,11}$ but, when exposed to air, it degrades almost instantly ${ }^{11}$.

Devices made from these materials can be protected from air by working in a glovebox and encapsulating them in a chemically inert material such as hexagonal boron nitride ${ }^{12,13}$. However, when developing these devices, it is often necessary to identify and analyze the flakes before encapsulation. This analysis requires either removing the sample from the inert environment of the glovebox or putting the analysis equipment in the glovebox. Removing the sample, even for a short time, risks damage via oxidation or hydration, while placing the necessary equipment inside a glovebox can be costly and cumbersome. To remedy this, we designed a hermetic transfer cell that safely encloses a sample, keeping it in an inert environment, so that it can be removed from the glovebox. While in the transfer cell, a sample sits $0.3 \mathrm{~mm}$ below a glass window to allow easy identification of flakes under a microscope as well as the use of optical analysis techniques such as photoluminescence or Raman spectroscopy.

Some two-dimensional materials, in addition to being air sensitive, are also difficult to exfoliate into thin flakes with the typical mechanical exfoliation method because a relatively high cleavage energy, relatively weak in-plane bonds, or both. Other methods, such as CVD growth ${ }^{14,15}$ liquid exfoliation ${ }^{16}$, or gold mediated exfoliation ${ }^{17,18}$ have been developed for producing thin layers but may result in less than pristine flakes and only work for certain materials. Although exfoliation of graphene at elevated temperatures has been known to produce large flakes for at least a decade ${ }^{19}$, this technique has been quantitatively characterized recently for both graphene and $\mathrm{Bi}_{2} \mathrm{Sr}_{2} \mathrm{CaCu}_{2} \mathrm{O}_{\mathrm{x}}$ flakes ${ }^{20}$. Here, we demonstrate that hot exfoliation improves exfoliation yield also for black phosphorus, a material that is notoriously difficult to exfoliate. This technique, together with a hermetic transfer cell, facilitates the exfoliation and analysis of air sensitive, two-dimensional materials. 


\section{Hot exfoliation of 2-D materials}

NOTE: This procedure is done inside a glovebox.

\section{Tape Preparation}

1. Cut a length of tape (see Table of Materials) that is $\approx 5-10 \mathrm{~cm}$ long and $\approx 2 \mathrm{~cm}$ wide. Place it, sticky side up, on the working area. Fold the ends of the tape for easier handling.

2. Using tweezers, deposit the desired material approximately one-quarter of the way down the length of the tape by repeatedly pressing the material into the tape.

3. Further distribute the material by folding the tape in half, sticking it to itself, and pulling it apart so that the material covers an area of at least $1 \mathrm{~cm}^{2}$. Depending on the material, repeat this multiple times: 1-2 times for black phosphorus, or several times for graphite or hexagonal boron nitride.

\section{Sample preparation}

1. Using the desired method, such as a carbide-tipped scribe, cleave an oxidized silicon wafer or other desired substrate into chips suitable for the experiment, $\leq 1 \mathrm{~cm}$ wide. Clean the chips by sonicating for $2 \mathrm{~min}$ in acetone, followed by isopropanol (IPA), at relatively low power (we used $12 \mathrm{~W}$ ). Blow the chips dry with $\mathrm{N}_{2}$.

2. Using the prepared tape, firmly press the deposited material onto the substrate. Apply firm pressure with a thumb or gently press with tweezers so the material contacts the chip as much as possible

3. Place the tape with substrate (substrate side down) on a hotplate at $120^{\circ} \mathrm{C}$ for $2 \mathrm{~min}$.

4. Allow the substrate to cool to RT and carefully remove it from the tape. Soak in acetone for 20 min to remove tape residue. Rinse with IPA for $30 \mathrm{~s}$ and dry the substrate with nitrogen. Depending on the material, further options for cleaning may be available, such as a forming gas anneal.

\section{Hermetic transfer cell construction, operation, and maintenance}

\section{Construction}

1. Construct the cell (Figure 2) out of the desired material (we used aluminum). It is $30 \mathrm{~mm}$ in diameter and $17.6 \mathrm{~mm}$ tall when closed. Fabrication drawings are available at http://churchill-lab.com/useful-things.

2. Make the base $16.2 \mathrm{~mm}$ tall with a raised sample platform that is threaded with $3 / 4-10$ threads with a vent cut into the threads. Where the cap meets the base, make an inset for an O-ring (see Table of Materials).

3. Make the cap $8.6 \mathrm{~mm}$ tall with matching female threads through the center.

4. Recess the cap by $0.2 \mathrm{~mm}$ to accommodate a $24 \mathrm{~mm}$ diameter $\times 0.1 \mathrm{~mm}$ thick coverglass window (here, borosilicate glass).

5. Apply a small amount of vacuum grease to the all sides of the O-ring and drop it into the base inset.

6. Before affixing the window to the cap of the cell, clean the cap in acetone and IPA to remove any oil or debris left by the machining process.

7. Attach the window to the cell cap using epoxy. Thoroughly mix the epoxy according to the manufacturer's specifications. Here, parts $A$ and $B$ are combined in a 1:1.8 ratio by weight.

8. Apply a small amount of epoxy to the recessed area on the cap and spread it around as evenly as possible.

9. Set a $0.1 \mathrm{~mm}$ thick, $24 \mathrm{~mm}$ diameter coverglass (borosilicate glass in this case) into the recess and gently press it into the epoxy. Ensure the window is level with the top of the cap and there that there are no bubbles in the epoxy.

10. Wipe up any extra epoxy so that nothing protrudes from the surface of the cap. Allow the epoxy to cure for the manufacturer prescribed time at room temperature.

\section{Operation}

NOTE: This procedure is done inside a glovebox.

1. Using the desired method, affix a prepared sample to the cell base (double sided tape, glue, etc.). The cell is designed to accommodate samples up to $1 \mathrm{~cm}$ wide and $0.7 \mathrm{~mm}$ thick, including the adhesive.

2. Firmly screw the cap onto the base. This makes a seal between the cap and base by compressing the O-ring. Make sure that the pressure inside the transfer cell does not exceed 3 mbar above the ambient pressure.

3. Check that the sample sits just below the window. The sample can now be safely removed from the glovebox.

\section{Window repair}

1. Using tweezers, remove any broken glass that is not firmly affixed to the epoxy. Break up what glass remains (using a carbide-tipped scribe or other method) so that the epoxy beneath is exposed.

CAUTION: Wear gloves and eye protection when removing broken glass.

2. Soak the cap in a 50:50 mix of acetone and trichloroethylene (TCE) for 1-2 h or until the epoxy softens and begins to separate from the cap. Rinse in IPA for $30 \mathrm{~s}$.

3. Peel off any loose epoxy and scrape the remaining epoxy from the surface with a razor blade. Take care not to damage the surface of the cap. Repeat the previous step if necessary.

4. Scrub the recessed area with acetone until the surface is clean of any epoxy residue. The cell window can now be replaced following the aforementioned steps. 


\section{Example uses of the transfer cell}

\section{Optical analysis}

1. For flake imaging, place the transfer cell under the microscope. The cell can be used with any conventional microscope. When focusing, be careful not to crash the objective into the fragile widow.

2. Proceed with desired method for finding material flakes.

\section{Polarized Raman spectroscopy}

1. For polarization-resolved Raman spectroscopy, align a laser spot to a flake of interest. In this case we use $633 \mathrm{~nm}$ wavelength and 50 $\mu \mathrm{W}$ power and a 100x objective lens. For black phosphorus, low laser power is required to prevent damage to the flake.

2. Using a half-wave plate, vary the polarization angle.

\section{Representative Results}

The goal of exfoliating two-dimensional materials is to isolate atomically thin layers. During the exfoliation process, flakes separate from the bulk crystal, leaving behind flakes of varying thicknesses, with a small probability for some flakes to be monolayers. By increasing the density and size of all exfoliated flakes, hot exfoliation increases the density and lateral size of thin flakes. This is accomplished by increasing the material area that makes close contact with the substrate. While in contact, gasses trapped between the material and the substrate expand during heating and are pushed out from under flakes. The removal of trapped gas allows more of the material to come into close contact with the substrate, thus increasing the amount of exfoliated flakes (Figure 1A,B) as clearly explained in Ref 20. Exfoliations of black phosphorus were performed using the typical mechanical exfoliation and hot exfoliation technique on silicon chips with $90 \mathrm{~nm}$ thick $\mathrm{SiO}_{2}$. By measuring the total area of deposited material on a $1 \mathrm{~cm} \times 1 \mathrm{~cm}$ silicon chip, it can be seen (Figure 1C) that hot exfoliation deposits 6-10 times more material. We note that in our experience other materials can be picked up from HF-cleaned substrates using polycarbonate following hot exfoliation, including graphene, hexagonal boron nitride, black phosphorus, $\mathrm{MnPSe}_{3}$, and $\mathrm{WSe}_{2}$. We used a 10:1 $\mathrm{HF}$ :water solution to clean the $\mathrm{SiO}_{2}$ substrates over a $15 \mathrm{~s}$ period. Note, $10 \% \mathrm{HF}$ etches $\mathrm{SiO}_{2}$ at a rate of $23 \mathrm{~nm} / \mathrm{min}^{21}$, so this process etches our substrates by $6 \mathrm{~nm}$.

We now consider the effectiveness of the hermetic transfer cell (Figure 3A) in maintaining an inert atmosphere when removed from a glovebox. $\mathrm{Crl}_{3}$ is particularly sensitive to hydration and degrades within seconds when exposed to air (Figure 3D). Inside a transfer cell, however, an exfoliated $\mathrm{Crl}_{3}$ sample remained unchanged for 15 hours (Figure 3B) and only began to show signs of degradation (blisters) after 24 hours (Figure 3C). While damage on a scale too small observe optically likely occurs on a shorter timescale, these results demonstrate that the hermetic transfer cell described here slows the sample degradation rate by at least three orders of magnitude (hours inside the cell compared with seconds outside).

To demonstrate use of the transfer cell for optical analysis of air-sensitive materials, we performed polarization-resolved Raman spectroscopy on a relatively thick $(>50 \mathrm{~nm}$ ) flake of black phosphorus (Figure 4A). The spectra were acquired using $50 \mu \mathrm{W}$ laser excitation at $632.8 \mathrm{~nm}$ with a $100 x$ objective lens. A half-wave plate was used to rotate the polarization of the excitation beam. In Figure 4B, three Raman peaks can be observed in BP at around 466, 438 and $361 \mathrm{~cm}^{-1}$, corresponding to $\mathrm{A}_{\mathrm{g}}{ }^{2}, \mathrm{~B}_{2 \mathrm{~g}}$ and $\mathrm{A}_{\mathrm{g}}{ }^{1}$ vibration modes respectively, regardless of the polarization, which agrees well with previous observations in bulk BP crystals for excitation and collection along the z-axis. ${ }^{5,22}$ The peak positions do not vary with polarization angle. However, the relative intensities of these three modes change significantly with incident light polarization. The vibration mode $\mathrm{A}_{\mathrm{g}}^{2}$, which has the strongest intensity variation with the excitation laser polarization, as shown in Figure $4 \mathrm{~B}, \mathrm{C}$, is associated with the atomic motion along the armchair direction. Therefore, as previously reported ${ }^{5}$, this vibration mode provides an effective method to determine the armchair direction of the BP crystal and hence the crystal orientation. In Figure 4C, the Raman intensity shows two maxima within one full rotation, located at $26.5^{\circ}$ and $206.5^{\circ}$ with respect to the $X$ and $Y$ axes defined in the microscope images, and we conclude that the armchair direction of the $\mathrm{BP}$ is oriented at $26.5^{\circ}$ for this flake. Similar optical spectroscopy methods can be used to determine crystal orientation and other properties, such as layer number or optical band gap, for other air-sensitive 2-D materials. 

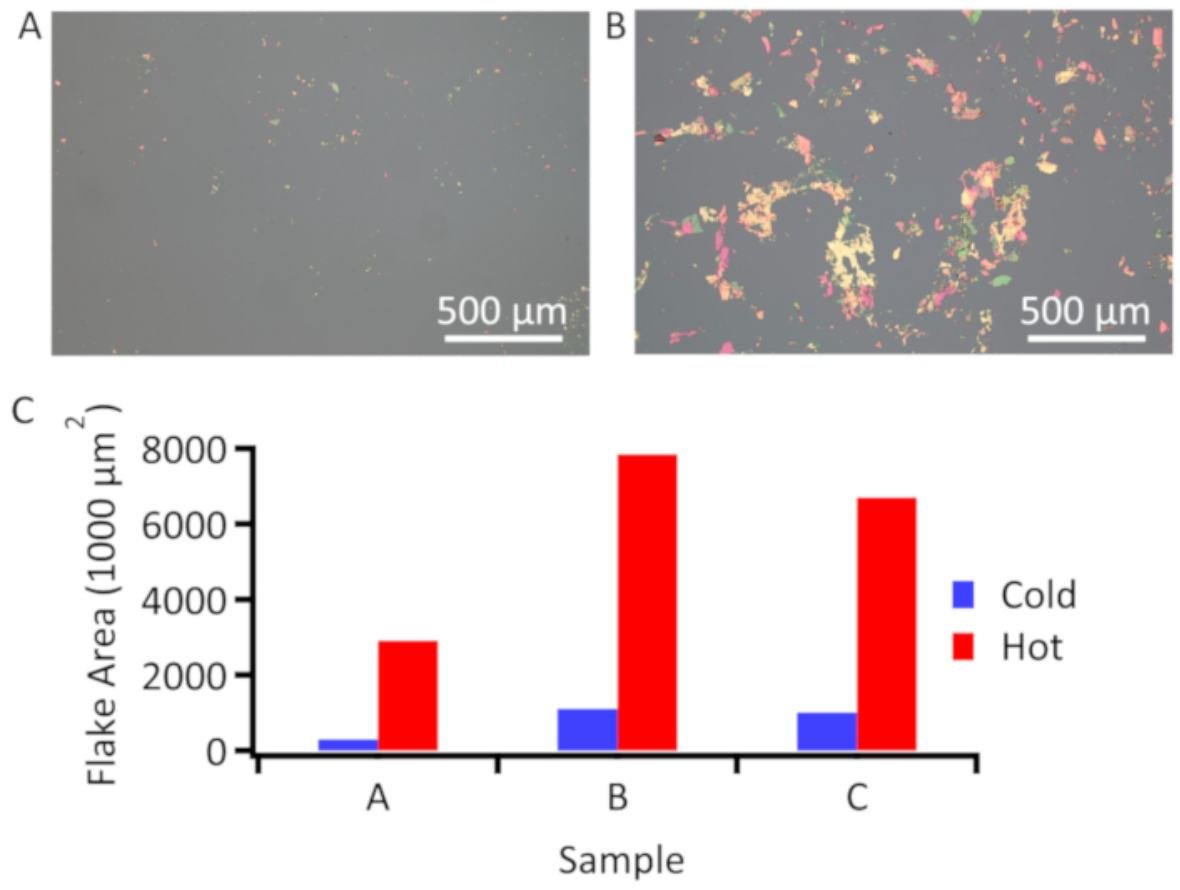

Figure 1: Distribution of material on an oxidized silicon chip. (A) Typical sample of black phosphorus exfoliated at room temperature. (B) Typical sample of black phosphorus exfoliated at $120^{\circ} \mathrm{C}$. (C) Histogram of exfoliated black phosphorus area using room temperature(cold) and hot exfoliation. Please click here to view a larger version of this figure.
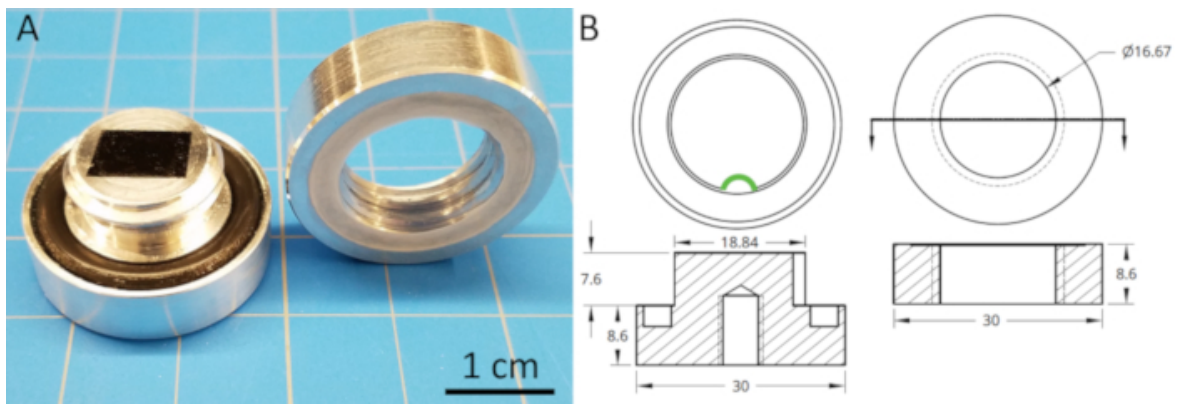

Figure 2: Transfer cell. (A) Picture of a hermetic transfer cell showing separate cap and base. (B) Schematic drawing of transfer. A vent (green) is cut into the threads. Note that the bottom of the base is tapped and threaded for mounting. Please click here to view a larger version of this figure. 

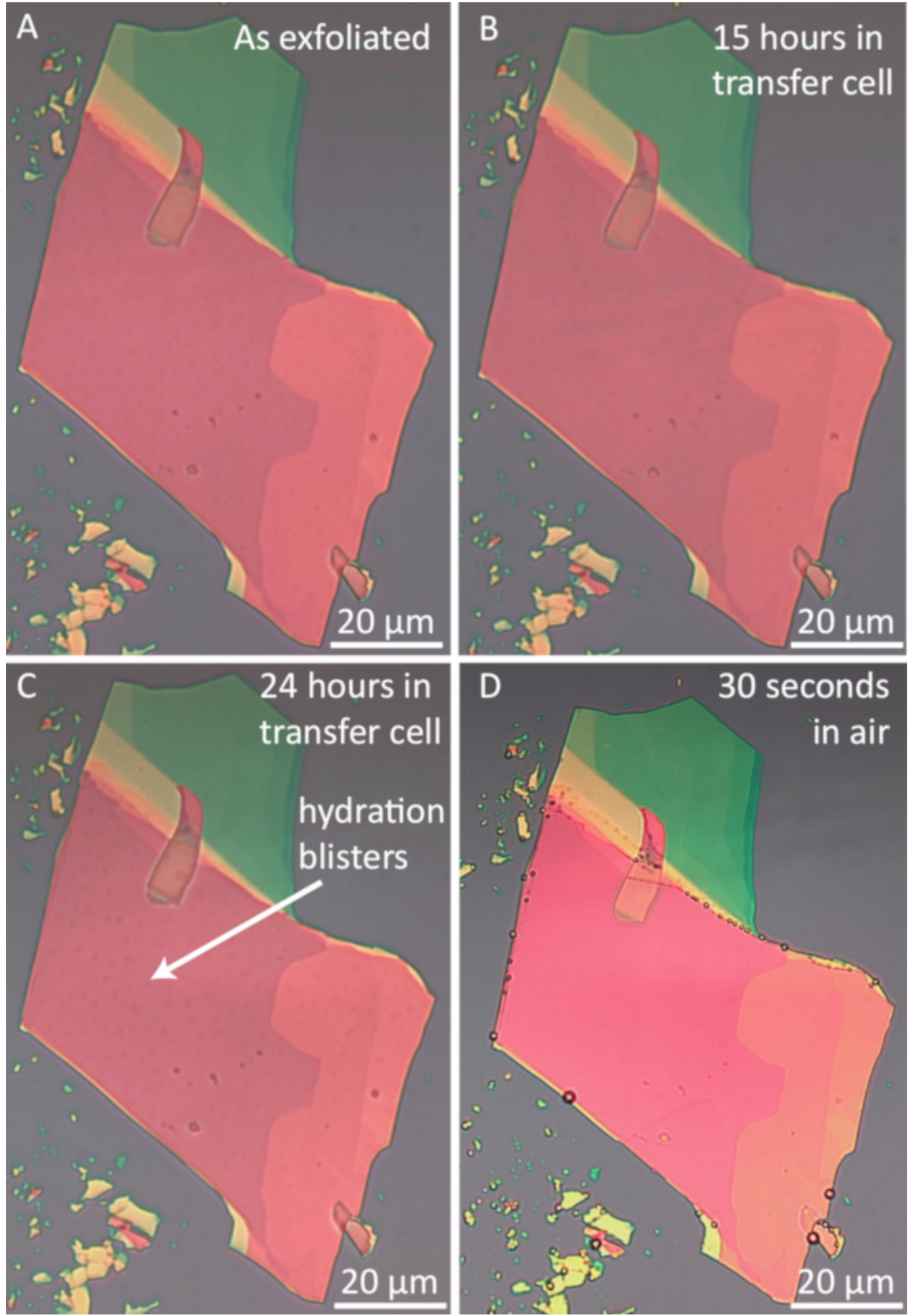

Figure 3: Transfer cell suppression of flake degradation. (A) Fresh $\mathrm{Crl}_{3}$ in a transfer cell (B) $\mathrm{Crl}_{3}$ in a cell after $15 \mathrm{~h}$. (C) $\mathrm{Crl}_{3}$ in a cell after 24 $\mathrm{h}$ Hydration blisters can be seen at this point. (D) $\mathrm{Crl}_{3}$ in air after $24 \mathrm{~h}$ in transfer cell and $30 \mathrm{~s}$ in air. Large areas of hydrated $\mathrm{Crl}_{3}$ have collected at the edges of the flake. Please click here to view a larger version of this figure. 

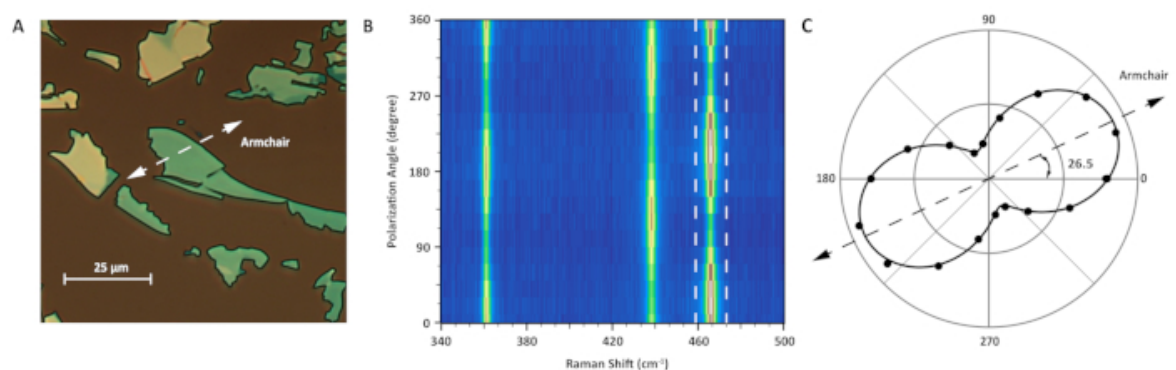

Figure 4: Crystalline orientation identification. (A) Optical micrograph of thick flakes of exfoliated BP. (B) Polarization-resolved Raman spectroscopy of a thick BP flake. (C) Polar plot of Raman intensity averaged over the spectral range in (B) as a function of linear excitation polarization angle (plot origin is zero intensity). The fit is a sine function plus a constant. The dashed line indicates the armchair direction. Please click here to view a larger version of this figure.

\section{Discussion}

Hot exfoliation retains the ability of typical mechanical exfoliation to produce pristine thin flakes while also avoiding many downfalls of alternatives. Like typical mechanical exfoliation, this technique is not limited to a small subset of materials. Hot exfoliation can be applied to any material that can be exfoliated using room-temperature mechanical exfoliation as long as the material tolerates heating to $120^{\circ} \mathrm{C}$ for $2 \mathrm{~min}$ in an inert atmosphere. We also note that it has been shown ${ }^{20}$ that the heating time and temperature (above $100{ }^{\circ} \mathrm{C}$ ) do not make any noticeable difference in flake density. Along with increased contact, average flake size can also be improved by increasing the bond strength between the substrate and the flakes. One way to do this would be by treating the substrate with $\mathrm{O}_{2}$ plasma but this would also make the flakes hard or impossible to pick up for use in devices requiring heterostructure fabrication ${ }^{20}$.

The transfer cell can be constructed from any suitable metal. We used aluminum because it is easy to machine but it should be noted that TCE (used to remove epoxy) is corrosive to aluminum when unstabilized, heated, or mixed with water. Stainless steel would be more durable and less reactive with TCE. However, we have not seen any corrosive effects using this method at RT. For imaging and analysis with high numerical aperture objectives, construction of the transfer cell is such that, when closed, the bottom of the window is $0.8 \mathrm{~mm}$ above the top of the base. With a $0.5 \mathrm{~mm}$ thick substrate and $0.1 \mathrm{~mm}$ thick adhesive, the sample sits $0.3 \mathrm{~mm}$ below the top of the transfer cell. This proximity allows for imaging and analysis with high magnification and relatively short working distance objectives. Exfoliated material can be clearly seen at 5 , 20,50 times magnification allowing for easy identification of thin flakes. At higher magnifications, spherical aberrations caused by the window significantly degrades the image quality. Provided that the sample substrate is less than $0.7 \mathrm{~mm}$ thick, there is no risk of over tightening the cell. When the cap is screwed down, excess gas is expelled through the vent in the threads. During construction, the precise location of the vent is not important, but it is important that it is not obstructed by the sample, vacuum grease or anything else. The vent prevents the fragile $0.1 \mathrm{~mm}$ thick window from breaking due to overpressure when the cap is screwed down. The window can only withstand pressure changes of a few mbar.

The coverglass window used for the transfer cells is made of borosilicate glass but for optical analysis at wavelengths other than visible to nearinfrared, other window materials may be use. For the best imaging, care should be taken when installing the glass window. If not seated properly, the distance between the sample and window could be larger than expected. Especially for small working distance objectives, this could cause the objective to crash into and break the window. Also, some epoxies will cure faster at higher temperatures, but because metals and glass have different thermal expansion coefficients, the widow will deform after cooling back to room temperature. The epoxy should be cured at the same temperature at which it will be used (i.e., if the cell will be used at room temperature), the epoxy should also be cured at room temperature.

\section{Disclosures}

The authors have nothing to disclose.

\section{Acknowledgements}

This work was supported by NSF award number DMR-1610126.

\section{References}

1. Koenig, S. P., Doganov, R. A., Schmidt, H., Castro Neto, A. H., Özyilmaz, B. Electric field effect in ultrathin black phosphorus. Applied Physics Letters. 104 (10), 103106 (2014).

2. Li, L. et al. Black phosphorus field-effect transistors. Nature Nanotechnology. 9 (5), 372-377 (2014).

3. Liu, H. et al. Phosphorene: an unexplored 2D semiconductor with a high hole mobility. ACS Nano. 8 (4), 4033 (2014).

4. Wang, X. et al. Highly anisotropic and robust excitons in monolayer black phosphorus. Nature Nanotechnology. 10 (6), 517 (2015).

5. Xia, F., Wang, H., Jia, Y. Rediscovering black phosphorus as an anisotropic layered material for optoelectronics and electronics. Nature Communications. 54458 (2014)

6. Castellanos-Gomez, A. et al. Isolation and characterization of few-layer black phosphorus. 2D Materials. 1 (2), 025001 (2014)

7. Island, J.O. et al. Environmental instability of few-layer black phosphorus. 2D Materials. 2 (1), 011002 (2015).

8. Huang, Y. et al. Interaction of Black Phosphorus with Oxygen and Water. Chemistry of Materials. 28 (22), $8330-8339$ (2016). 
9. Gong, C. et al. Discovery of intrinsic ferromagnetism in two-dimensional van der Waals crystals. Nature. 546 (7657), 265 (2017).

10. Huang, B. et al. Layer-dependent ferromagnetism in a van der Waals crystal down to the monolayer limit. Nature. 546 (7657), 270 (2017).

11. Lado, J. L., Fernández-Rossier, J. On the origin of magnetic anisotropy in two dimensional Crl3. 2D Materials. 4 (3), 35002 (2017).

12. Li, X., Yin, J., Zhou, J., Guo, W. Large area hexagonal boron nitride monolayer as efficient atomically thick insulating coating against friction and oxidation. Nanotechnology. 25 (10), 105701 (2014).

13. Liu, Z. et al. Ultrathin high-temperature oxidation-resistant coatings of hexagonal boron nitride. Nature Communications. 4, 2541 (2013).

14. Li, X. et al. Large-Area Synthesis of High-Quality and Uniform Graphene Films on Copper Foils. Science. 324 (5932), 1312-1314 (2009).

15. Sutter, E. A., Flege, J.-I., Sutter, P. W. Epitaxial graphene on ruthenium. Nature Materials. 7 (5), $406-411$ (2008).

16. Lotya, M. et al. High-yield production of graphene by liquid-phase exfoliation of graphite. Nature Nanotechnology. 3 (9), $563-568$ (2008).

17. Magda, G. Z. et al. Exfoliation of large-area transition metal chalcogenide single layers. Scientific reports. 5, 14714 (2015).

18. Desai, S. B. et al. Gold-Mediated Exfoliation of Ultralarge Optoelectronically-Perfect Monolayers. Advanced materials (Deerfield Beach, Fla.). 28 (21), 4053-4058 (2016).

19. Williams, J. R. Electronic transport in graphene: $p$-n junctions, shot noise, and nanoribbons. ProQuest Dissertations Publishing (2009).

20. Huang, Y. et al. Reliable Exfoliation of Large-Area High-Quality Flakes of Graphene and Other Two-Dimensional Materials. ACS Nano. 9 (11), 10612-10620 (2015).

21. Williams, K. R., Muller, R. S. Etch rates for micromachining processing. Journal of Microelectromechanical Systems. 5 (4), $256-269$ (1996).

22. Ribeiro, H. B., Pimenta, M. A., de Matos, C. J. S. Raman spectroscopy in black phosphorus. Journal of Raman Spectroscopy. 49 (1), $76-90$ (2018). 